\title{
Collective effects on mass asymmetry in fission*
}

\author{
J. A. Maruhn \\ Oak Ridge National Laboratory, Oak Ridge, Tennessee 37830 \\ W. Greiner \\ Institut für Theoretische Physik der Universität Frankfurt, West Germany
}

(Received 22 September 1975)

\begin{abstract}
The development of the mass asymmetry vibrations in the final stages of the fission process is studied with an approximate treatment of the coupling to relative motion. A parametrized friction is introduced and its effects are studied. Numerical results are presented for ${ }^{236} \mathrm{U}$, together with estimates for the kinetic energy of the fragments.
\end{abstract}

$\left[\begin{array}{c}\text { RADIOACTIVITY, FISSION }{ }^{236} \mathrm{U} \text {; calculated mass distribution, kinetic energy distribution. } \\ \text { Collective dynamics, shell correction method, cranking model. }\end{array}\right]$

Even though the calculation of potential energy surfaces based on the shell-correction method ${ }^{1}$ has been very successful in explaining the basic properties of fissioning nuclei, ${ }^{2}$ it has been argued that a knowledge of the potential energy surface alone is not sufficient to predict the dynamical behavior of the system. ${ }^{3}$ Thus, it was shown that extremely varying mass parameters can produce effects similar to those of a secondary minimum in the potential and distort the wave functions in practically any respect. ${ }^{4}$ It seems of great importance to investigate the magnitude of the effects which may be caused not by an arbitrary mass parameter with rather extreme properties, but by one which may reasonably be expected for collective motion during the fission process. Such mass parameters may be obtained from the cranking model $l^{5}$ using the wave functions of a phenomenological two-center shell model which also gives the potential energy surface. This approach will be followed in this paper.

Mass parameters obtained in this way have been calculated for a variety of two-center models, ${ }^{6,7}$ and, in general, they show rather large oscillations due to the varying shell and pairing structures as a function of the collective parameters. Peak-to-valley ratios of more than 10 to 1 are not infrequent. Since the computation of the mass parameters tends to be quite laborious, however, one has to be content with relatively few points, so that the resulting functions must be interpolated with a considerable degree of uncertainty.

Knowing the collective potential energy and the mass parameters, in principle, allows the calculation of all dynamical properties of the systemaside from certain possible ambiguities in the quantization. Because of the large amount of computation involved and the nonexistence of readily usable codes for that purpose, computations have been carried out by reducing the problem to one collective coordinate only ${ }^{8}$ or computing the onedimensional WKB penetration probability through the fission barrier along any path in a multidimensional potential energy surface. ${ }^{7}$ The penetration probability is then given by

$$
P=\exp \left[-\frac{2 m}{\hbar} \int\left(\sum|E-V| B_{x_{i} x_{j}} \dot{x}_{i} \dot{x}_{j}\right)^{1 / 2} d t\right],
$$

with $x_{i}=x_{i}(t)$ as parametrization of a path in the space of $N$ collective coordinates $x_{i}$. By varying the path, one may search for that one which has highest probability and take that probability as the real multidimensional one. Although this method is only an approximation to a real multidimensional WKB theory, ${ }^{9}$ it already yields some interesting results, showing, e.g., that the mostfavorable path need not even pass close to the saddle point of the static potential energy surface, which had until then been considered to be of such eminent importance in fission theory. This shows again that the potential energy surface alone does not give reliable information on the fission process.

A different type of caiculation was carried out in Ref. 8. There the dynamical behavior of one particular collective coordinate, viz. the mass asymmetry, was studied in a one-dimensional approximation, i.e., neglecting all effects due to coupling with other collective degrees of freedom. It was shown that the zero-point vibrations in this degree of freedom occurring during the post-tunneling stage of fission between saddle point and scission are qualitatively similar to the observed mass distributions. The approximations were certainly too restrictive to expect more quantitative results, but several important character- 
istics emerged. It could be seen that the potential energy surface still seemed to determine the main behavior of the wave functions, with the strongly oscillating mass parameter influencing the details such as peak-to-valley ratio, spread, and fine structure. Since the behavior of mass asymmetry did not vary negligibly with the position on the fission path, however, a one-dimensional treatment did not seem completely adequate.

These two problems will be investigated in a more detailed manner in the present paper.

We have extended the calculations described in Ref. 7 to take the relative motion of the nascent fragments and its interactions with the mass asymmetry vibrations into account, following the dynamical evolution of the latter for some part of the descent from saddle point to scission. In this way the development of the asymmetry vibrations can be studied more realistically and the results should also shed some light on the general problem of the influence of variable collective mass parameters in fission.

For the calculations we utilized the asymmetric two-center shell model. ${ }^{10}$ Of its five independent shape parameters, only the two of interest in this context were retained, namely the mass asymmetry

$$
\xi=\frac{A_{1}-A_{2}}{A_{1}+A_{2}},
$$

with the fragment masses $A_{1}$ and $A_{2}$ related to the geometric size of the nascent fragments, and the elongation $\lambda$, which is defined as the total length of the deformed nucleus in units of the diameter of a spherical nucleus of equal mass. A specimen shape is shown in Fig. 1. The remaining collective parameters were determined by requiring minimum potential energy for a given pair $(\lambda, \xi)$. As mentioned, the potential energy is calculated by the shell-correction method, and the collective mass parameters are obtained from the cranking model.

The classical total energy of the system is given by

$$
H(\lambda, \xi)=\frac{1}{2} B_{\lambda \lambda} \dot{\lambda}^{2}+B_{\lambda \xi} \dot{\lambda} \dot{\xi}+\frac{1}{2} B_{\xi \xi} \dot{\xi}^{2}+V(\lambda, \xi) .
$$

Before quantizing Eq. (3), some simplifications have to be made. First, as was already shown in Ref. 7 and turned out to be true in all later calculations as well, the coupling mass $B_{\lambda \xi}$ may be neglected. Secondly, we want to deal with the behavior of the nucleus shortly after it has finished tunneling through the fission barrier (this proceeds mainly in the $\lambda$ degree of freedom), so that it starts its motion along $\lambda$ with zero kinetic enerby, i.e., $\dot{\lambda}=0$.

Recent calculations ${ }^{11}$ have shown that the system

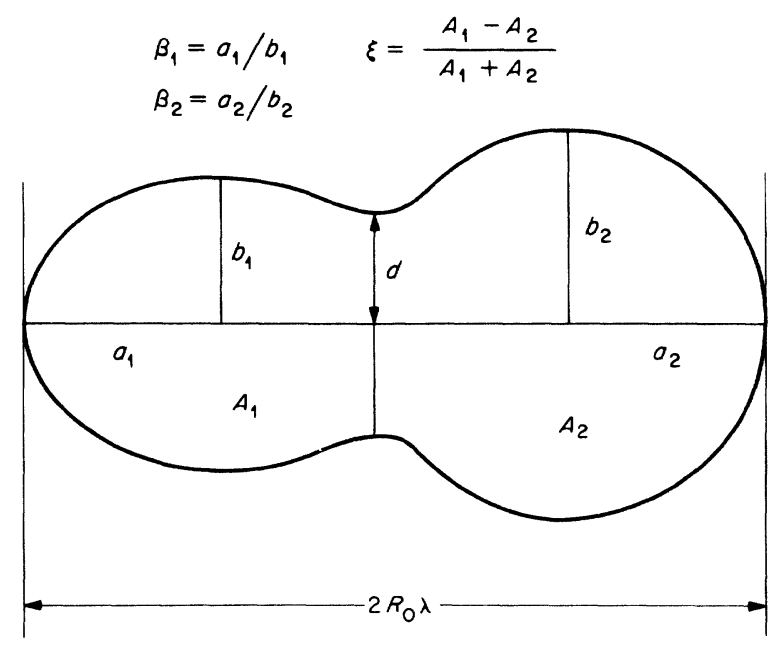

FIG. 1. Parametrization of the nuclear surface in the asymmetric two-center shell model (Ref. 10). The free parameters are the elongation $\lambda$, the deformations $\beta_{1}$ and $\beta_{2}$, the mass asymmetry $\xi$, and a quantity determining the neck size $d$.

will be in the ground state of $\xi$ at the exit point even if there was some excitation before tunneling. During the acceleration phase we shall set no restriction on the velocity $\dot{\lambda}$, so that all cases from slow adiabatic motion up to the "sudden" case can be treated.

Using these approximations, the $\xi$-dependent part of Eq. (3) can be quantized while $\lambda$ is regarded as a parameter:

$\left[-\frac{\hbar^{2}}{2 \sqrt{B}} \frac{\partial}{\partial \xi} \frac{1}{\sqrt{B}} \frac{\partial}{\partial \xi}+V(\xi, \lambda)\right] \phi_{\nu}(\xi, \lambda)=E_{\nu}(\lambda) \phi_{\nu}(\xi, \lambda)$,

with $B$ an abbreviation for $B_{\xi \xi}$. The quantization procedure follows Pauli and Podolsky, ${ }^{12}$ so that we get a volume element in $\xi$ involving $B$, as seen from the orthonormality conditions

$$
\int \phi_{\nu}^{*}(\xi, \lambda) \phi_{\mu}(\xi, \lambda)[B(\xi)]^{1 / 2} d \xi=\delta_{\mu \nu} .
$$

The solutions of Eq. (4), the adiabatic basic functions $\phi_{\nu}(\xi, \lambda)$, were discussed in Ref. 7. Here, however, we are interested in the additional $\lambda$ dependence as well. So we set up the total wave function as a sum over the $\phi_{\nu}$ with $\lambda$-dependent coefficients

$\Psi(\xi, \lambda)=\sum_{\nu} a_{\nu}(\lambda) \phi_{\nu}(\xi, \lambda) \exp \left[-\frac{i}{\hbar} \int_{0}^{t} E_{\nu}\left(\lambda\left(t^{\prime}\right)\right) d t^{\prime}\right]$.

If $\lambda$ is determined classically as a function of time, $\lambda=\lambda(t)$, Eq. (6) is nothing else but the usual ansatz for solving the time-dependent Schrödinger 
equation.

The classical equation of motion for $\lambda$ may be obtained by calculating the average value of the energy (3) in the wave function (6), replacing the kinetic energy of $\xi$ and the potential energy by the expectation value of the $\xi$ Hamiltonian as appearing in Eq. (4), whereas the coupling mass $B_{\lambda \xi}$ is again neglected. This yields

$$
\langle H\rangle_{\mathrm{av}}=\frac{1}{2}\left\langle B_{\lambda \lambda}\right\rangle \dot{\lambda}^{2}+\sum_{\nu}\left|a_{\nu}(\lambda)\right|^{2} E_{\nu}(\lambda),
$$

with the $\xi$-averaged mass parameter

$$
\begin{aligned}
& \left\langle B_{\lambda \lambda}\right\rangle=\sum_{\nu, \mu} a_{\nu}^{*}(\lambda) a_{\mu}(\lambda) \\
& \quad \times \int \phi_{\nu}^{*}(\xi, \lambda) B_{\lambda \lambda}(\lambda, \xi) \phi_{\mu}(\xi, \lambda) \sqrt{B} d \xi .
\end{aligned}
$$

The second term in Eq. (7) is the effective potential for relative motion; it depends on the excitation of higher states in $\xi$ and thus on the velocity $\dot{\lambda}$ and, in general, on the previous history of the system. Thus, it contains the effects of excitation during $\lambda$ motion, which may be regarded as friction. However, this is not the usual type of friction commonly related to the excitation of singleparticle states, but a purely collective one (fragmentation friction), which nonetheless should also be present in reality. It should be noted that this effective potential needs no renormalization, because the potential $V(\xi, \lambda)$ of Eq. (4) which determines the $E_{\nu}(\lambda)$ and thus $V(\lambda)$ is already renormalized.

Another interesting consequence of Eq. (7) is that even if there is no excitation at all in $\xi$, i.e., if $a_{\nu}(\lambda)=\delta_{\nu 0}$ throughout, the potential still contains the effects of a varying zero-point energy in $\xi$, which reflects the changes in the potential and mass parameter as $\lambda$ increases. So in the presence of a varying zero-point energy, one-dimensional calculations of the WKB type ${ }^{7}$ may not be accurate. However, in the present calculations, it turned out that the effects of both zero-point energy and excitation energy in the $\xi$ degree of freedom were less than $0.5 \mathrm{MeV}$ in magnitude and thus negligible compared to the overall drop of $V(\xi, \lambda)$ of about $10 \mathrm{MeV}$ in the range of $\lambda$ values concerned.

The time development of the coefficients $a_{\nu}(\lambda)$ may as usual be obtained by inserting the wave function of Eq. (6) into the time-dependent Schrödinger equation

$$
i \hbar \frac{\partial}{\partial t} \Psi=\left[-\frac{\hbar^{2}}{\sqrt{B}} \frac{\partial}{\partial \xi} \frac{1}{\sqrt{B}} \frac{\partial}{\partial \xi}+V\right] \Psi(\xi, \lambda(t))
$$

to yield

$$
i \hbar \dot{a}_{\nu}=\sum_{\mu} \hbar \dot{\lambda}\left\langle\nu\left|\frac{\partial}{\partial \lambda}\right| \mu\right\rangle \exp \left[\frac{i}{\hbar} \int\left(E_{\nu}-E_{\mu}\right) d t\right] .
$$

The pair of equations (7) and (10) determine the dynamical behavior completely. Starting with a given initial set of $a_{\nu}$, one may calculate the average mass $\left\langle B_{\lambda \lambda}\right\rangle$ and the potential force in Eq. (7) via Eq. (8). Because the force depends on the rate of change of the $a_{\nu}$ 's, and these in turn are determined by the force, there is a requirement of selfconsistency between these two equations. This can be fulfilled approximately by first computing the force under the assumption of constant $a_{\nu}$ 's, and then adjusting it with the actually calculated ones. This problem, however, proved to be of practically no significance so that, in general, a simple explicit time-stepping algorithm could be used.

In order to estimate the possible effects of dissipation into single-particle degrees of freedom, we have included a parametrized frictional force in the calculation by replacing the requirement of constant total energy $H$ by

$$
\frac{d H}{d t}=-f \dot{\lambda}^{2}
$$

with $f$ the coefficient of friction.

The results presented below refer to the fission of ${ }^{236} \mathrm{U}$. Figure 2 shows the potential energy surface along that part of the "fission path" we have investigated. $\lambda=1.65$, the starting point, corresponds to approximately the exit point for spontan-

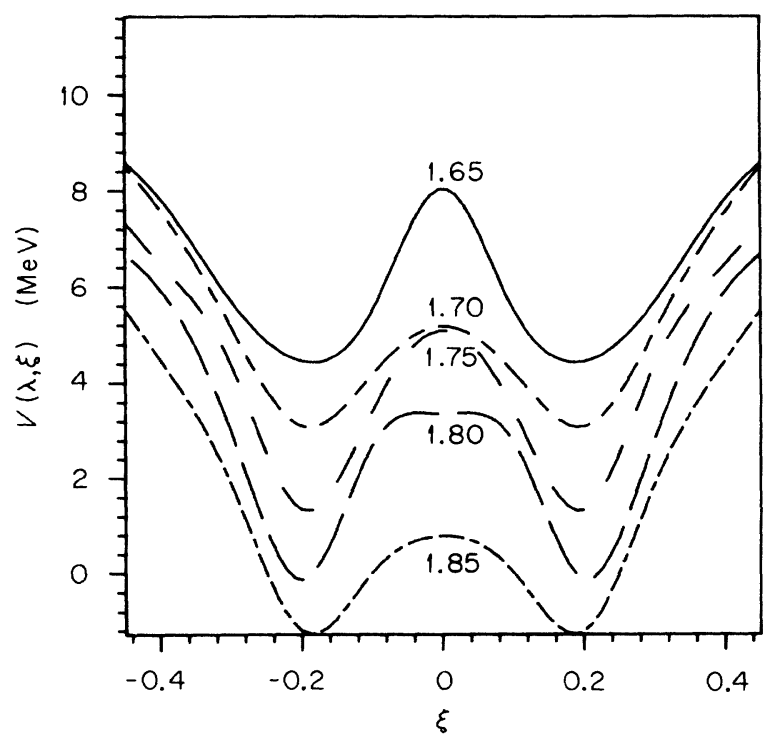

FIG. 2. The collective potential energy surface as a function of asymmetry $\xi$, for various values of the elongation $\lambda$ as indicated at the curves. 
eous fission, and $\lambda=1.85$ is the (somewhat arbitrary) termination point for the calculation. There does not seem to be much to be gained in following the system further down the slope, because the ultimate rapid formation of a neck changes the collective motion drastically, although it probably does not change the fragment masses to any large extent anymore. ${ }^{13}$ The figure shows that the potential drops down uniformly in this range of $\lambda$ values, but with the dependence on $\xi$ still changing considerably.

The mass parameters $B_{\xi \xi}$ and $B_{\lambda \lambda}$ are shown in Figs. 3 and 4 . They show the well-known oscillatory behavior with the details not well determined because of the relatively small number of points (11) used for each curve. Nevertheless, two interesting gross features may be seen in the curves. The average value of $B_{\lambda \lambda}$ decreases with $\lambda$ increasing, coming closer to the limiting value

$$
B_{\lambda \lambda}(\lambda \rightarrow \infty)=\left(2 R_{0}\right)^{2} B_{\text {red }}(\xi),
$$

i.e., the reduced mass of the fragmentation $\xi . R_{0}$ is the radius of the spherical nucleus and the factor is caused by the difference in definition between $\lambda$ and the relative distance. On the other hand, the average value of $B_{\xi \xi}$ rises with $\lambda$ increasing, showing that the exchange of mass between the fragments is becoming increasingly hindered by the formation of a neck. This problem has been investigated in Ref. 13 .

Figures 5 and 6 show the results for the dynamical development of the collective wave function, obtained in the manner discussed above.

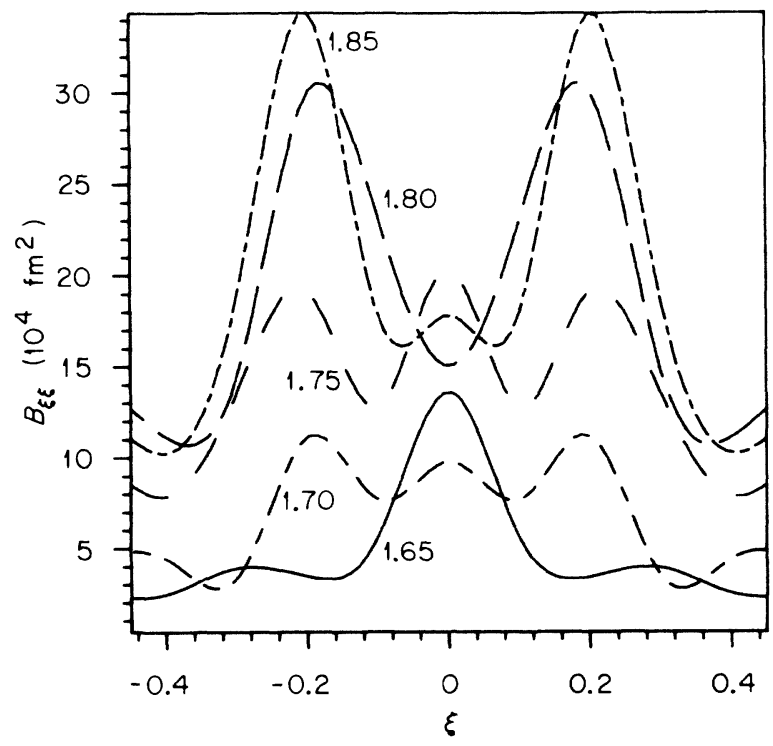

FIG. 3. The mass parameter $B_{\xi \xi}$ for asymmetry oscillation as a function of $\xi$ for different values of $\lambda$.

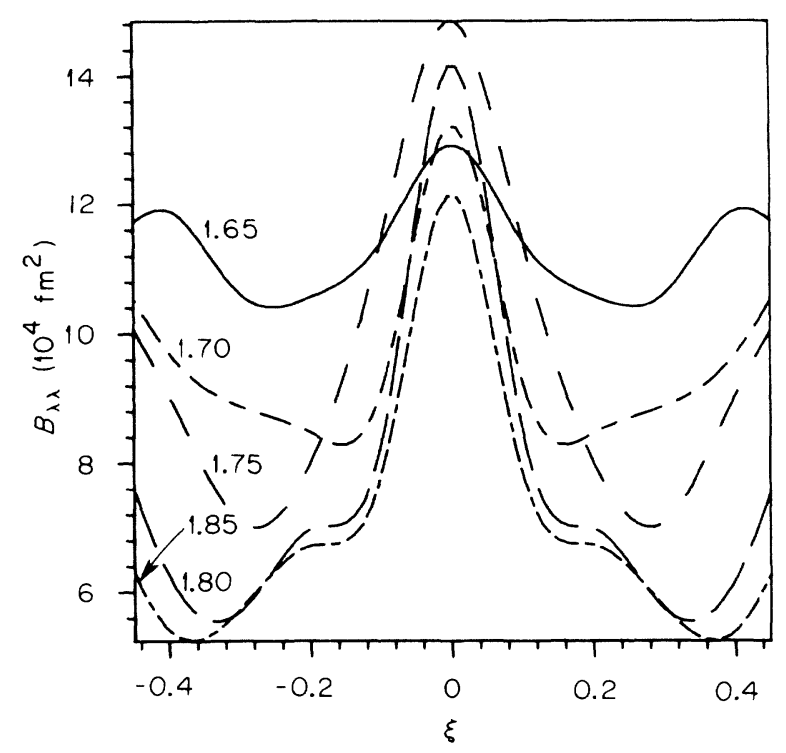

FIG. 4. The mass parameter $B_{\lambda \lambda}$ for relative motion as a function of $\xi$ for different values of $\lambda$.

The calculation was done for several values of the friction coefficient $f$, which were chosen such as to show the transition from unimpeded acceleration to very slow motion. This can be seen almost immediately from the plot of collective velocities $\dot{\lambda}$ in Fig. 5.

Much of the collective behavior depends critically on the velocity $\lambda$ and thus on friction. Only

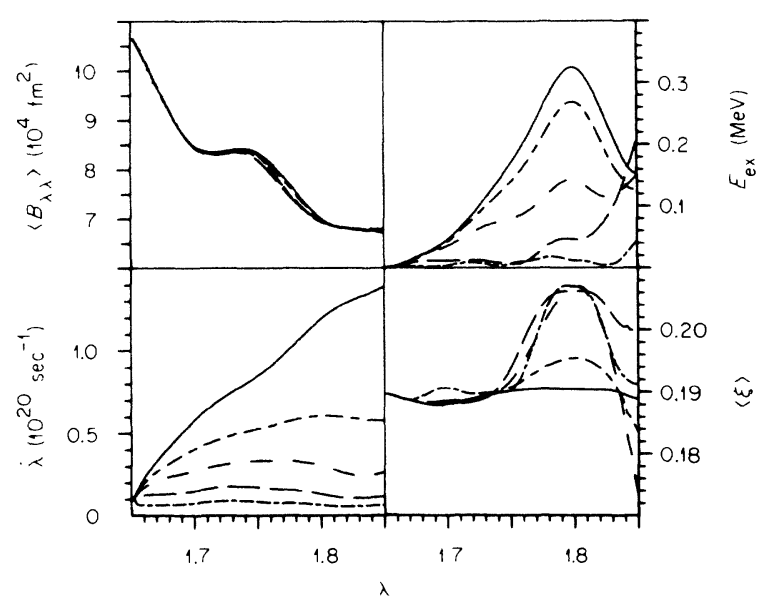

FIG. 5. Results of the dynamical calculation for varying friction parameters given below. Upper left: average $B_{\lambda \lambda}$-mass parameter; lower left: relative velocity $\dot{\lambda}$; upper right: collective excitation energy ; lower right: average mass asymmetry $\langle\xi\rangle$. All are plotted as functions of elongation. The dash patterns associated with the friction values can be seen in the plot of $\lambda$, where $f$ takes the values $f=0,5,10,20$, and 40 (in units of $10^{-19} \mathrm{MeV} \mathrm{sec}$ ) from the highest down to the lowest curve in that order. 


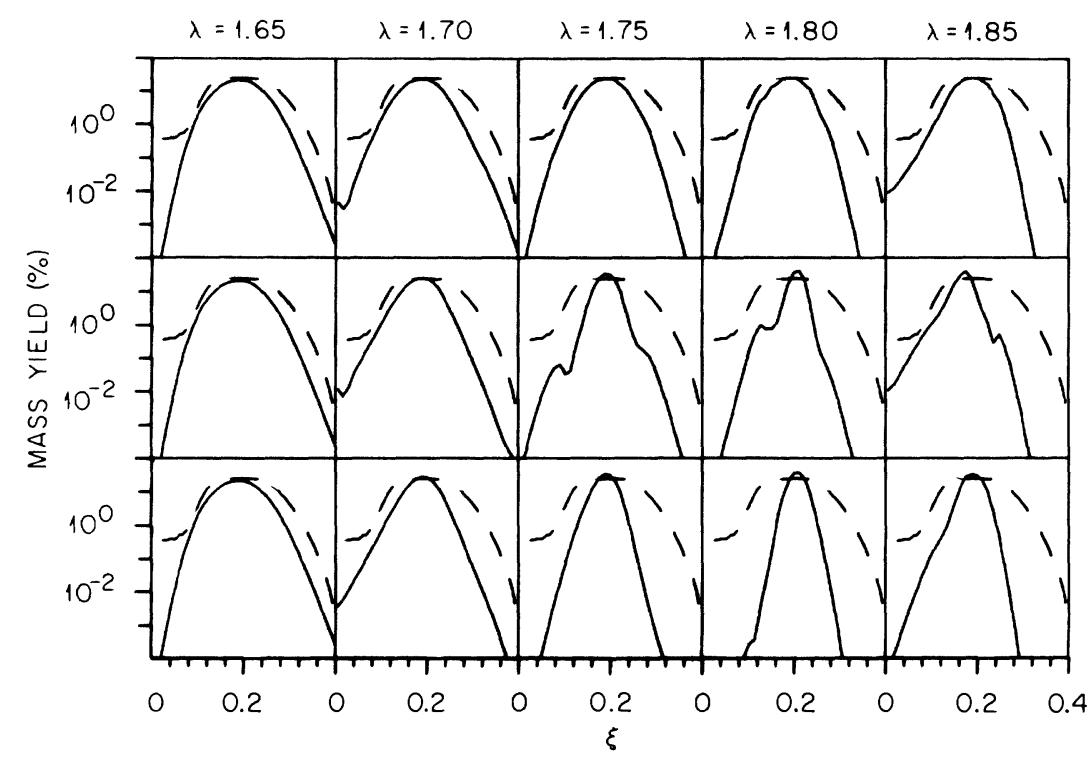

FIG. 6. The collective wave functions, converted to an equivalent mass yield. From left to right the curves pertain to $\lambda=1.65,1.7,1.75,1.8$, and 1.85 , so that each row shows the development of the wave function in a particular case. The top row is for friction $f=0$, the middle one for $f=10^{-18} \mathrm{MeV} \mathrm{sec}$, and the bottom one for $f=4 \times 10^{-18} \mathrm{MeV} \mathrm{sec}$.

the average mass $\left\langle B_{\lambda \lambda}\right\rangle$, calculated similarly to Eq. (9), does not seem to be sensitive. It only drops down rather smoothly to the limiting value of Eq. (13) for the most probable asymmetry.

The collective excitation energy has been defined as

$$
E_{\mathrm{ex}}(\lambda)=\sum_{\nu}\left|a_{\nu}\right|^{2}\left(E_{\nu}-E_{0}\right) .
$$

It depends quite strongly on friction. For a very slow "adiabatic" movement, it remains quite small and conversely gets largest for the "rapid" case with no friction. In the latter case the wave function does not change much with $\lambda$ and the excitation simply reflects the fact that the $\xi$ wave function at the start is really an excited state for later values of $\lambda$ with their different potentials and mass parameters. The fact that $E_{\text {ex }}$ goes down near $\lambda=1.85$ can be explained by observing that there the potential and mass become more similar to those near $\lambda=1.65$ again, so that the $\xi$ wave function is closer to the ground state.

The collective probability distribution

$$
\Psi^{*}(\xi, \lambda) \Psi(\xi, \lambda)=\sum_{\nu, \mu} a_{\nu}^{*}(\lambda) a_{\mu}(\lambda) \phi_{\nu}^{*}(\xi, \lambda) \phi_{\mu}(\xi, \lambda)
$$

is shown in Fig. 6 for three different values of the friction. For no friction the wave function changes little so that we seem to be close to the "sudden" case, whereas for very strong friction and slow descent, it adapts to the potential and mass pa- rameter at each stage, and, in general, tends to become narrower because of the increasing average value of $B_{\xi \xi}$. The intermediate cases show some oscillations which are due to coherent excitation of higher $\xi$ states.

One of the most interesting quantities to be calculated from these wave functions is the mean asymmetry defined as

$$
\langle\xi\rangle=\sum_{\nu, \mu} a_{\nu}^{*} a_{\mu}\langle\nu\|\xi\| \mu\rangle .
$$

It is also shown in Fig. 5. Apparently its behavior depends quite strongly on friction so that we have a clear demonstration of the influence of dynamics. On the other hand, the range of $\xi$ values covered in this plot corresponds to a difference in fragment mass of only about four units, so that the dynamic effects are there, but not dominant. The location of the minimum in the potential energy surface still provides a good first approximation to the preferired asymmetry. On the other hand, the spread of the probability distribution around the maximum seems to depend much more sensitively on dynamics.

For comparison, we did the calculation with no frictional force also under the assumption of a constant $\lambda$ mass, to see if the variations in this mass were of any large importance. The results are shown in Figs. 7 and 8. The values of $B_{\lambda \lambda}$ selected covered the actual range of the variable mass. Since a large $B_{\lambda \lambda}$ mass tends to slow down 


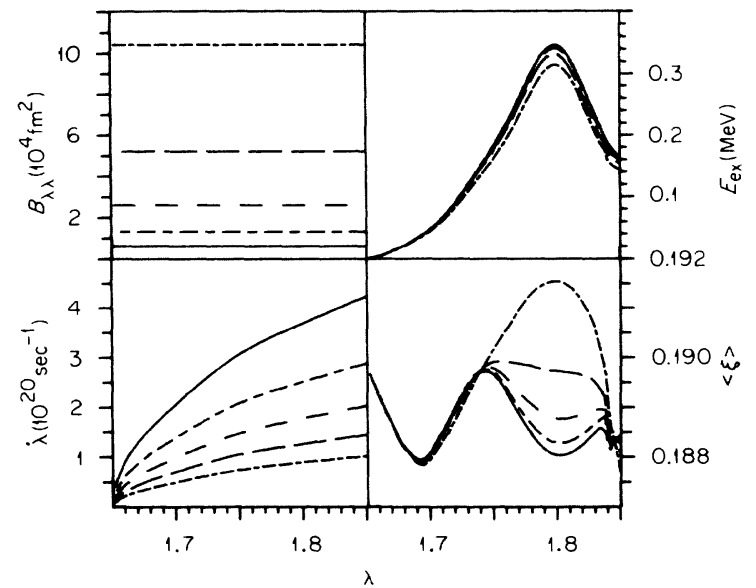

FIG. 7. The same quantities as in Fig. 5 , plotted for constant $B_{\lambda \lambda}$ mass, the values of $B_{\lambda \lambda}$ for each family of curves being shown in the upper left.

motion in $\lambda$, its effects are similar to those of an increased friction, but on the whole the dependence of the velocity and excitation on $\lambda$ seems to be smoother than with $B_{\lambda \lambda}$ varying. Also, the spread of $\langle\xi\rangle$ values is much reduced, amounting to only a change of about one unit in the fragment masses. It seems that the stronger dependence of $\langle\xi\rangle$ on $\lambda$ is the most prominent effect of having $B_{\lambda \lambda}$ varying.

Figures 9 and 10 show the effects of a nonzero initial velocity $\dot{\lambda}$. Apparently the results are not changed appreciably, the noticeable differences in $\langle\xi\rangle$ being exaggerated by a small scale. This,

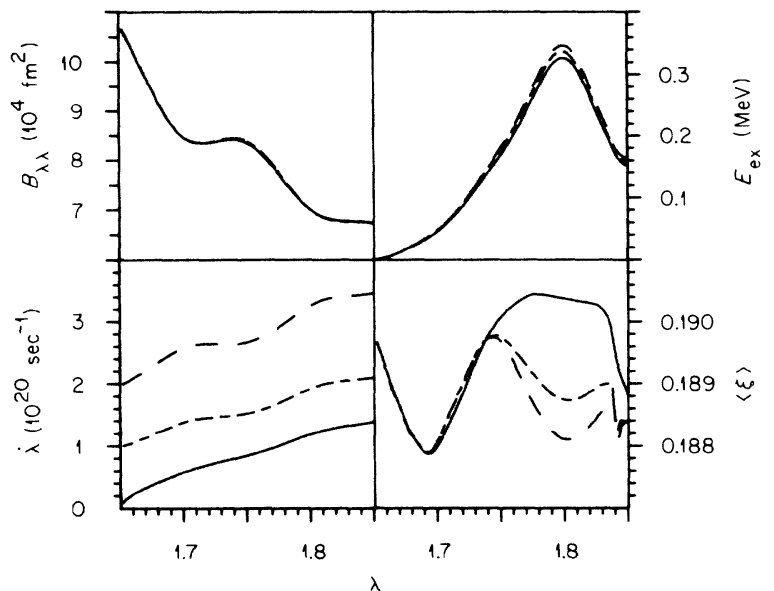

FIG. 9. The same quantities as in Fig. 5, plotted for different initial speeds $\dot{\lambda}$. The initial values can be seen in the lower left.

together with the behavior of the wave functions in Fig. 10, indicates that the situation is close to the "sudden" case,${ }^{14}$ which is characterized by an almost constant wave function becoming independent of the changes of the potential and mass parameter with $\lambda$. The results of the calculation come close to this limit except for the low probability region in the mass distribution, where numerical accuracy becomes important.

Summarizing the results of the calculations, we have obtained some insight into the behavior of a collective system with two coupled degrees of freedom exemplified by the interplay of mass

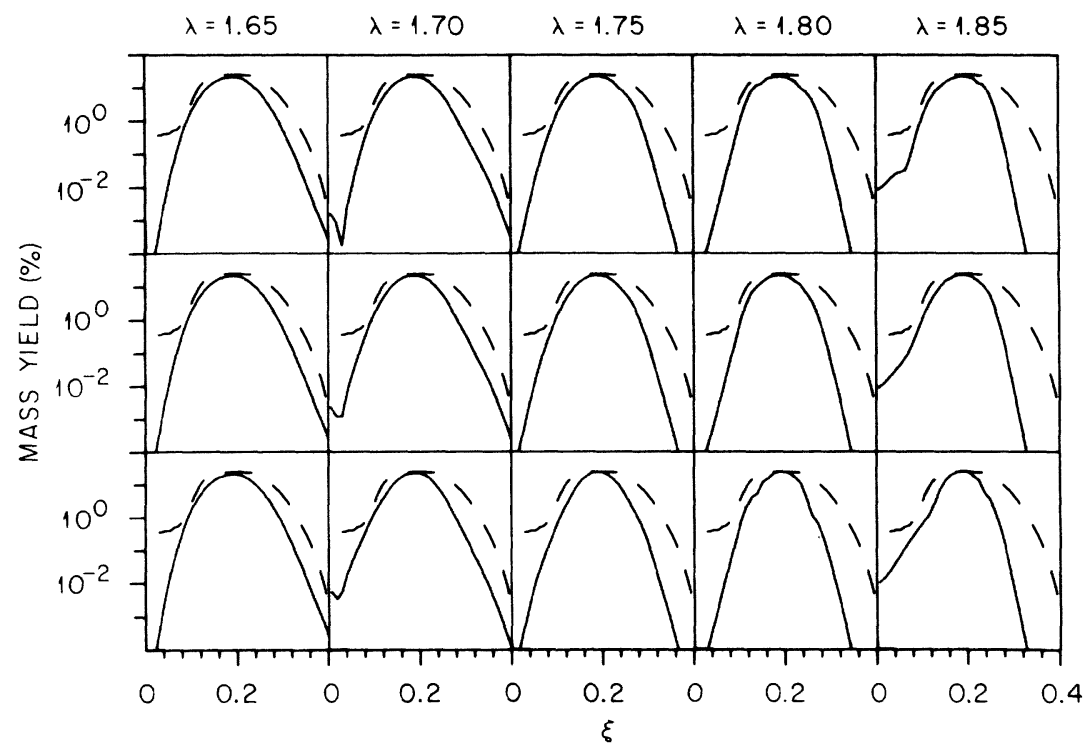

FIG. 8. Wave functions for constant $B_{\lambda \lambda}$. The top row corresponds to $B_{\lambda \lambda} / M=10^{6} \mathrm{fm}^{2}$, the middle row to $2.5 \times 10^{5}$ $\mathrm{fm}^{2}$, and the bottom row to $6 \times 10^{4} \mathrm{fm}^{2}$. 


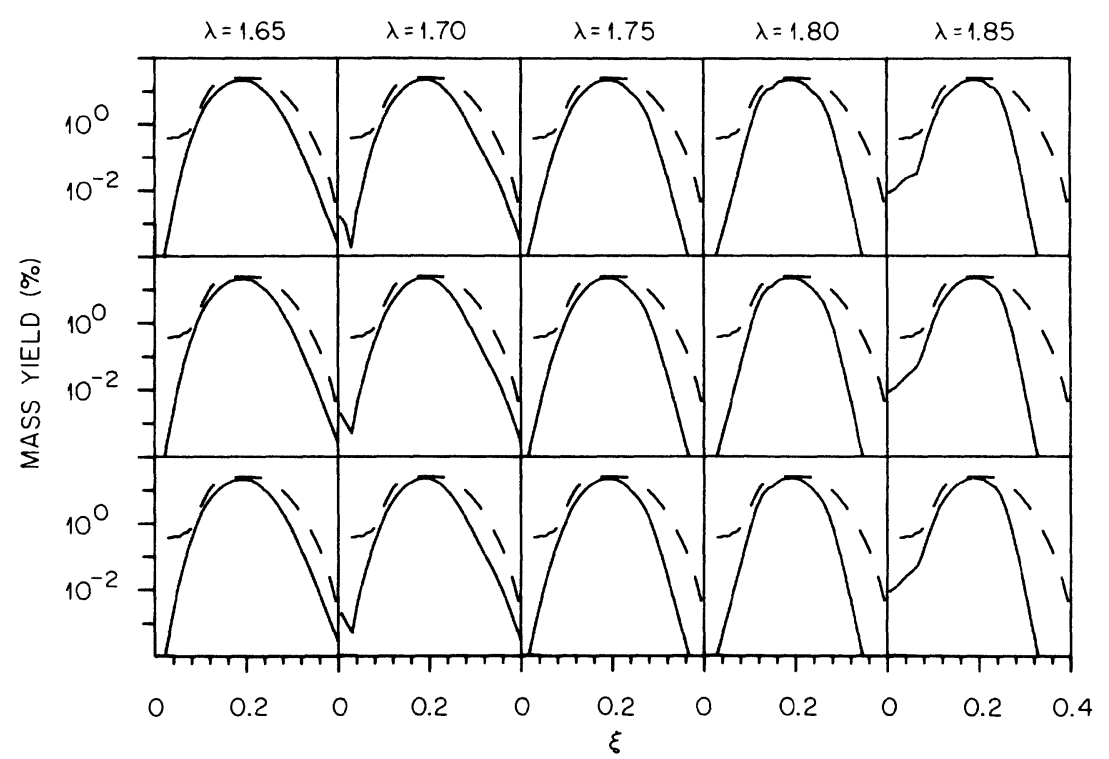

FIG. 10. Wave functions for different initial velocities. Top row : $\dot{\lambda}_{\text {init }}=0$; middle row: $\dot{\lambda}_{\text {init }}=10^{20} \mathrm{~s}^{-1}$; bottom row: $\dot{\lambda}_{\text {init }}=2 \times 10^{20} \mathrm{~s}^{-1}$.

asymmetry and relative motion in fission. It was seen that the static potential energy surfaces are quite sufficient to determine the gross features of fission and that the detailed behavior of the mass parameters does not change the dynamics as drastically as is possible in theory. ${ }^{4}$ However, any detailed study of the peak-to-valley ratio and the structure of the mass distribution curve will not be possible without studying the dynamics. These details are still beyond the reach of any theory of this type because of the intrinsic restrictions made in the shape parametrization and the choice of a single-particle potential, but we may hope that constrained Hartree-Fock calculations may carry us farther.

If we assume for the moment that the potential energy surface and mass parameters calculated in the two-center shell model ${ }^{10}$ are sufficiently realistic, some further deductions may be made from these results. In this case, the results seem to show that the theoretical mass distribution comes closest to the experimental one for a rather rapid descent with no friction or even some initial velocity. Now the smallest coefficient of friction used may be converted to a viscosity by dividing by the nuclear volume, in order to obtain an order of magnitude estimate:

$$
\eta=\frac{5 \times 10^{-19} \mathrm{MeV} \mathrm{sec}}{\frac{4}{3} \pi r_{0}^{3} \mathrm{~A}} \approx 0.5 \mathrm{TP} .
$$

This seems to be somewhat larger than theoretical estimates lying in the range of 0.01 to 0.1 TP. ${ }^{15,16}$ Accordingly, if the theoretical estimates are believed, the real system behavior should be rather close to the sudden case with viscous effects relatively unimportant. This is true only, however, as long as only collective dynamics are considered. The single-particle heating of the nucleus described by viscosity will change the potential energy surface and the mass parameters, ${ }^{17}$ so that these arguments are only preliminary, especially since single-particle heating will tend to make the potential shallower and thus counter the collective effects of slowing as seen in Fig. 6. If the internal excitation is estimated to be about $20 \mathrm{MeV}$, the nuclear temperature of $0.9 \mathrm{MeV}$ should be just in the range where the potential energy surface changes drastically according to Ref. 17. Thus, heating effects will have to be included in these considerations; nevertheless, it is clear how fission mass distributions may, in principle, help in the determination of nuclear viscosity by fitting a coefficient of friction or viscosity to the experimental distributions.

Another problem which could be investigated by comparison with experiment is the question of whether friction is of the simple functional form assumed in Eq. (11), or whether some higher power of $\dot{\lambda}^{2}$ or an even more complicated functional form should be used. In order to check the sensitivity of the results to the assumed $\lambda$ dependence of friction, we did some calculations using a form proportional to $\dot{\lambda}^{4}$, as suggested by Schütte and Wilets. ${ }^{18}$ Some results are shown in Fig. 11. It appears that the development of the 


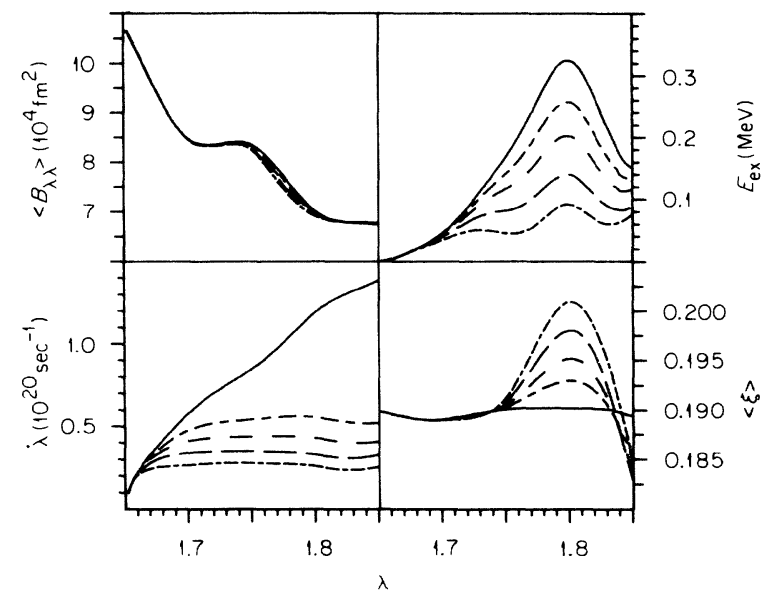

FIG. 11. The same quantities as in Fig. 5, plotted for varying values of a friction of the form $-g \dot{\lambda}^{4}$ replacing the right-hand side of Eq. (11). The values of the coefficient $g$ are $g=0,5,10,40$ (in units of $10^{-19} \mathrm{MeV} \mathrm{sec}^{3}$ ), pertaining to the highest down to the lowest curve in the $\lambda$ plot, in that order.

collective velocity with time is characteristically different; the transition from the acceleration phase to an asymptotic almost constant velocity is much more rapid. So the region where the sudden case is reached and the final distribution is practically determined may be shifted considerably. In the present case, however, the distributions show roughly similar variations as in Fig. 6 and have thus been omitted for brevity. Clearly the final distribution will be very sensitive to the details of the acceleration only if the potential surface shows major variations in the area traversed.

Finally, we tried to get a very simple estimate of the kinetic energy distribution as a function of fragment mass. For that, we assumed that the fragment deformations $\beta_{1}$ and $\beta_{2}$ do not change any more from $\lambda=1.85$ to the scission point. If the simplified four-parameter shapes of Mosel and Schmitt ${ }^{19}$ are used, the scission-point shapes are defined completely by $\xi, \beta_{1}(\xi), \beta_{2}(\xi)$, and $\lambda$ determined so that the fragments touch. We estimate the kinetic energy by subtracting the Coulomb energy of two separate fragments with deformation $\beta_{1}$ and $\beta_{2}$ from the Coulomb energy of the touching configuration. It has to be assumed in this model that the charge to mass ratio $Z / A$ is uniform and applies to the fragments as well.

The relative kinetic energy $\frac{1}{2}\left\langle B_{\lambda \lambda}\right\rangle \dot{\lambda}^{2}$ is included in the results, but it is independent of fragment mass in the framework of the present theory.

The results are shown in Table I. They have an overall shift of more than $40 \mathrm{MeV}$ compared
TABLE I. Fragment kinetic energies for fission of ${ }^{236} \mathrm{U}$. For each asymmetry $\xi$, the corresponding heavy fragment mass $A_{H}$, deformations $\beta_{1}$ and $\beta_{2}$ and theoretical and experimental kinetic energies are given. The experimental results are interpolated from the measurements of Ref. 20. The shifted theoretical values contain an additive constant to make them agree with experiment at $\xi=0$.

\begin{tabular}{llccccc}
\hline \hline$\xi$ & \multicolumn{1}{c}{$A_{H}$} & $\beta_{1}$ & $\beta_{2}$ & $E_{\text {kin }}$ & $\begin{array}{c}E_{\text {kin }} \\
\text { (shifted) }\end{array}$ & $\begin{array}{c}E_{\text {kin }} \\
\text { (exp.) }\end{array}$ \\
\hline 0.0 & 118 & 1.01 & 1.01 & 197.8 & 154 & 154 \\
0.1 & 129.8 & 0.90 & 1.10 & 210.6 & 166.8 & 180 \\
0.2 & 141.6 & 1.00 & 1.22 & 191.1 & 147.3 & 170 \\
0.3 & 153.4 & 1.02 & 0.90 & 200.7 & 156.9 & 156 \\
0.4 & 165.2 & 1.00 & 1.00 & 178.7 & 134.9 & $\cdots$ \\
0.5 & 177 & 0.95 & 0.98 & 151.3 & 107.5 & $\cdots$ \\
\hline \hline
\end{tabular}

to the experiment, which is probably caused by the fact that Mosel's parametrization does not allow for an independent variation of neck size and elongation, so that the scission-point configuration may not be sufficiently elongated. This problem could be tackled in the five-parameter parametrization used for the other calculations presented in this paper, but this would require a more extensive dynamical calculation to compute the rate of necking-in as a function of elongation. We present the results of the simple calculation nonetheless, because the main features of the dependence of kinetic energy on mass division should be caused by deformation in the nascent fragments, so that the dependence on $\xi$ should be more reliable than the overall absolute values.

However, if we renormalize the theoretical values by an additive constant so that they agree with experiment at the symmetric point, it appears that some trends are reproduced. The dip for symmetric fission is there and the value at $\xi=0.3$ agrees surprisingly well. On the other hand, the value at $\xi=0.2$ is off quite considerably. So the results seem encouraging but are certainly not yet quantitatively comparable to experiment.

It is to be expected that more can be learned about the kinetic energies if the dynamical calculation is carried through to the scission point. This, however, would require a dynamic treatment of the neck size as well, since close to scission the potential energy may not have a definite minimum as a function of neck size, so that the usual method of minimization to replace a coordinate by a fixed value does not work any more.

On the other hand, before this is attempted it seems more urgent to study the behavior of the collective dynamics under the influence of single- 
particle heating, to see how the potential energy surfaces and mass distributions are modified by internal excitation. The failure to include this is the major shortcoming of the pure collective the- ory presented here and such a calculation should enhance its value considerably and perhaps bring quantitative comparison with experiment within reach.
*Research sponsored by the U.S. Energy Research and Development Administration under contract with Union Carbide Corporation, by the Bundesministerium fir Forschung and Technologie, and by the Gesellschaft für schwerionenforschung (GSI).

${ }^{1}$ V. M. Strutinsky, Nucl. Phys. A95, 420 (1967); A122, 1 (1968).

${ }^{2}$ M. Brack, J. Damgaard, A. S. Jensen, H. C. Pauli, V. M. Strutinsky, and C. Y. Wong, Rev. Mod. Phys. 44, 320 (1972).

${ }^{3}$ L. Wilets, Theories of Nuclear Fission (Oxford U.P., London and New York, 1964).

${ }^{4}$ H. Hofmann, Z. Phys. 250, 14 (1972).

${ }^{5}$ S. T. Belyaev, K. Dan. Vidensk. Selsk. Mat.-Fys. Medd. 31, No. 11 (1959). For a systematic discussion, particularly of the nonadiabatic effects in the cranking model, see S. Liran, H. J. Scheefer, W. Scheid, and W. Greiner, Nucl. Phys. A248, 191 (1975).

${ }^{6}$ P. Lichtner, D. Drechsel, J. Maruhn, and W. Greiner, Phys. Rev. Lett. 28, 829 (1972).

${ }^{7}$ T. Ledergerber and H. C. Pauli, Phys. Lett. 39B, 307 (1972).

${ }^{8} \mathrm{P}$. Lichtner, D. Drechsel, J. Maruhn, and W. Greiner, Phys. Lett. 45B, 175 (1973); J. Maruhn and W. Greiner, Phys. Rev. Lett. 32, 548 (1974).

${ }^{9}$ W. H. Miller, J. Chem. Phys. 53, 1949, 3578 (1970).

${ }^{10} \mathrm{~J}$. Maruhn and W. Greiner, Z. Phys. 251, 431 (1972).

${ }^{11}$ H. Massmann, P. Ring, and J. O. Rasmussen, Lawrence Berkeley Laboratory Report No. LBL-4023 (un- published).

${ }^{12} \mathrm{~W}$. Pauli, in Handbuch der Physik (Springer-Verlag, Berlin, 1933), Vol. 24, p. 120; B. Podolsky, Phys. Rev. 32, 812 (1928).

${ }^{13}$ H.-J. Fink, J. Maruhn, W. Scheid, and W. Greiner, Z. Phys. 268, 321 (1974).

${ }^{14}$ See, e.g., A. B. Migdal and V. P. Krainov, Approximation Methods in Quantum Mechanics (Benjamin, New York, 1969).

${ }^{15}$ K. T. R. Davies, S. E. Koonin, J. R. Nix, and A. J. Sierk, Los Alamos Report No. LA-UR-75-5 (unpublished).

${ }^{16} \mathrm{R}$. Wieczorek, R. W. Hasse, and G. Stissmann, in Proceedings of the Third International Atomic Energy Agency Symposium on the Physics and Chemistry of Fission, Rochester, 1973, (International Atomic Energy Agency, Vienna, 1974), Vol. 1, p. 523.

${ }^{17}$ M. G. Mustafa, University of Maryland, Department of Physics \& Astronomy Technical Report No. 74-098, 1974 (unpublished).

${ }^{18} \mathrm{G}$. Schütte and L. Wilets, in Proceedings of the Third International Atomic Energy Agency Symposium on the Physics and Chemistry of Fission, Rochester, 1973 (see Ref. 16, Vol. 1, p. 503.

${ }^{19}$ U. Mosel and H. W. Schmitt, Phys. Rev. C $\underline{4}, 2185$ (1971).

${ }^{20}$ H. W. Schmitt, J. H. Neiler, and F. J. Walter, Phys. Rev. 141, 1146 (1966). 